\title{
Inhibition of tankyrase 1 in human gastric cancer cells enhances telomere shortening by telomerase inhibitors
}

\author{
HAO ZHANG* ${ }^{*}$, MENG-HUA YANG ${ }^{*}$, JING-JING ZHAO, LING CHEN, SONG-TAO YU, \\ XU-DONG TANG, DIAN-CHUN FANG and SHI-MING YANG
}

Institute of Gastroenterology, Southwest Hospital, Third Military Medical University, Chongqing 400038, P.R. China

Received March 30, 2010; Accepted June 8, 2010

DOI: 10.3892/or_00000955

\begin{abstract}
Telomere stability is believed to be related to aging and tumorigenesis. Besides telomerase, telomere length is also regulated by several telomere-specific binding proteins. Tankyrase 1, a telomeric poly(ADP-ribose) polymerase (PARP), elongates telomere length by inhibiting TRF1 binding to telomeres. In order to study the synergistic action of tankyrase 1 and telomerase in the maintenance of telomere length in mammalian cells, we constructed antisense tankyrase 1 (aTNKS) eukaryotic expression vectors and then transfected them into the SGC-7901 human gastric cancer cell line, as well as SGC-7901 cells that had been transfected with antisense hTR (7901-ahTR) and antisense hTERT (7901-ahTERT) with DOTAP liposomes. The activity of telomerase, telomere length and telomerase-associated protein activities were measure by TRAP-ELISA, Southern blot and Western blot analysis, respectively, in aTNKS transfected and untransfected cells. The results demonstrated that telomere length was significantly shorter in cells with concomitant tankyrase 1 and telomerase inhibition than by either tankyrase 1 or telomerase inhibition alone, in SGC7901 cells. We also found that aTNKS had no effect on telomerase activity. These results reveal that inhibition of tankyrase 1 could shorten telomere length and play a synergistic role with telomerase inhibitors in telomere length shortening in the SGC-7901 gastric cancer cell line. Co-inhibition of tankyrase 1 and telomerase activity may be a rational strategy for telomere-directed gastric cancer therapeutics.
\end{abstract}

Correspondence to: Dr Shi-Ming Yang or Dr Dian-Chun Fang, Institute of Gastroenterology, Southwest Hospital, Third Military Medical University, Chongqing 400038, P.R. China

E-mail: shimingyang@yahoo.com

E-mail: fangdianchun@hotmail.com

*Contributed equally

Abbreviations: hTERT, human telomerase reverse transcriptase; hTR, human telomerase RNA; TP1, human telomerase associated protein 1; TRF, terminal restriction fragment; TRAP, telomeric repeat amplification protocol

Key words: tankyrase 1, telomerase, telomere, hTERT, TRF1, gastric cancer

\section{Introduction}

Telomeres are nucleoprotein complexes at the ends of linear eukaryotic chromosomes that are essential for chromosome stability and integrity (1-3). In humans, they are composed of repetitive non-coding DNA sequences (5'-TTAGGG-3') that are approximately 5-20 kb in length. Telomeres are maintained by telomerase, a reverse transcriptase that adds telomeric repeats to the chromosome ends (4). Several components of human telomerase have been identified, including the RNA component (hTR) (5), telomerase associated protein 1 (TP1) (6) and telomerase reverse transcriptase (hTERT) (7). In most normal human somatic tissues, telomerase is repressed, and as a result, shortening of the telomeres has been observed. When telomere shortening reaches a critical length, telomeres lose their ability to protect chromosome ends, resulting in chromosomal degradation and fusion. In contrast to normal somatic human cells, immortalized cells (including cancer cells) and germ cells express telomerase $(8,9)$ and keep their telomere length. In these cells, telomeres are regulated by a homeostatic mechanism that includes telomerase and telomere-binding proteins (10). There are 2 major telomerebinding proteins: TRF1 (telomere repeat binding factor 1) and TRF2 (telomere repeat binding factor 2) (11). Both proteins act in cis as a negative regulator of telomere length by controlling the access of telomerase to telomeres. This is achieved directly or through the interaction of TRF1 and TRF2 with other proteins (12-15).

The enzyme tankyrase 1, a telomeric poly(ADP-ribose) polymerase (PARP), was identified as a component of the human telomeric complex (13). It inhibits TRF1 from binding with telomeres through the tankyrase-mediated ADP-ribosylation of the TRF1 protein (13). It has been confirmed that tankyrase 1 acts as a positive regulator of telomere length (16). Unlike telomerase, tankyrase 1 is ubiquitously expressed and distributed in all somatic cells. Although tankyrase 1 has no recognizable nuclear localization signal (NLS), it is present in both the nucleus and cytoplasm $(10,17,18)$. The nuclear localization of tankyrase 1 requires an interaction with TRF1, which contains an NLS (16). Overexpression of NLS-tagged tankyrase 1 in telomerase-positive cells releases TRF1 from telomeres and induces telomere elongation (16). Seimiya et al reported that tankyrase 1 diminished the effects of telomerase inhibitors in telomerase-positive cells, suggesting that tankyrase 1 counteracted the effects of telomerase inhibitors 


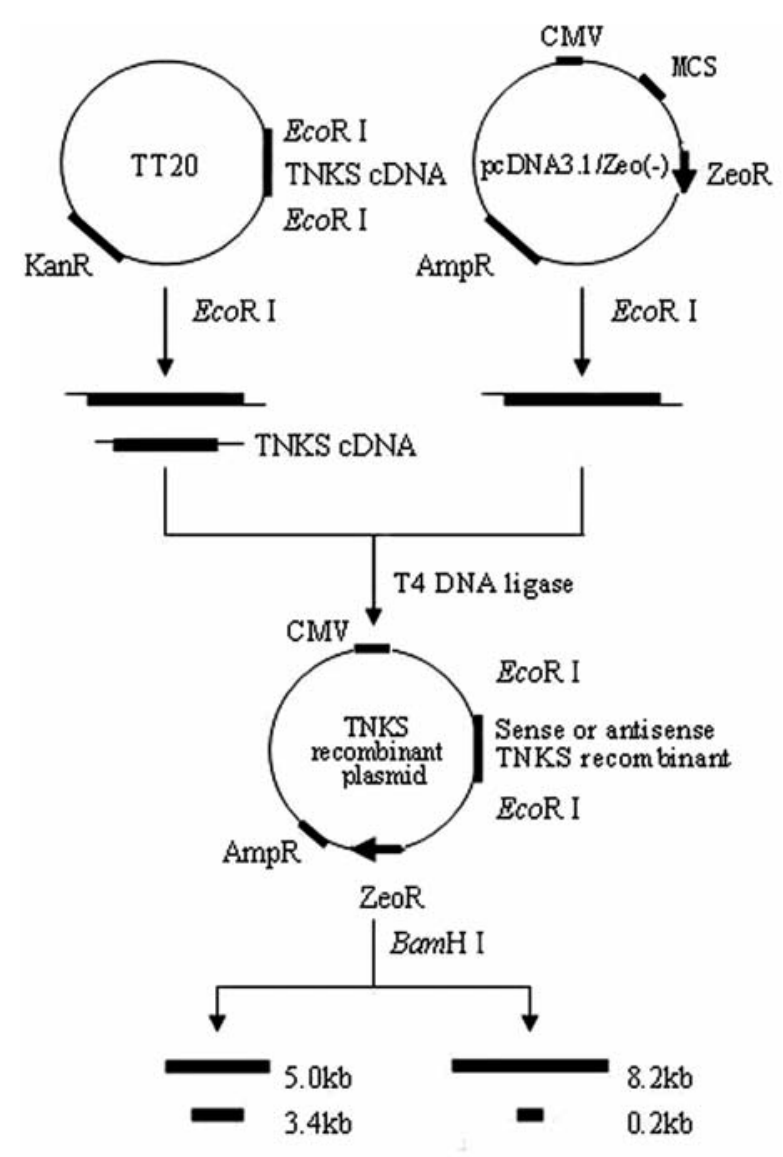

Sense TNKS recombinant Antisense TNKS recombinant

Figure 1. Strategy and procedure of the construction of the antisense tankyrase 1 eukaryotic expression vector.

on telomere length by facilitating the access of residual telomerases to telomeres (19). However, there is no evidence that tankyrase 1 directly regulates telomerase activity.

In our previous studies, we have confirmed that both telomerase activity and telomere length are decreased in the human gastric cancer cell line SGC-7901 transfected with antisense-hTERT (ahTERT) and antisense-hTR (ahTR) $(20,21)$. In this study, we report that telomere length is significantly shorter in SGC-7901 cells when tankyrase 1 and telomerase are both inhibited, compared to SGC-7901 cells where tankyrase 1 or telomerase is inhibited alone. These results suggest that tankyrase 1 , as well as telomerase, may be a rational target for telomere-directed cancer therapeutics.

\section{Materials and methods}

Construction of antisense Tankyrase 1 recombinant plasmids. The TT20 plasmid which contains the full length complementary DNA (cDNA) of tankyrase 1 was a gift from Professor Susan Smith and Titia de Lange from Rockefeller University, New York, NY, USA. The tankyrase $1 \mathrm{cDNA}$ was cloned between XhoI and EcoRI site of the plasmid. The construction procedures are shown in Fig 1. In brief, tankyrase 1 cDNA was digested with XhoI/EcoRI from the TT20 plasmid and then inserted into the EcoRI site of the eukaryotic pcDNA3.1/Zeo(-) expression vector (Invitrogen,
USA). The orientation of the recombinant DNA was further confirmed by BamHI restriction endonuclease analysis and sequencing. The sense and antisense tankyrase 1 recombinant vectors were named pcDNA-sTNKS and pcDNA-aTNKS, respectively.

Cell culture and DNA transfection. SGC-7901 cells transfected with an antisense gene of human telomerase RNA (7901-ahTR) (21), an antisense gene of the human telomerase reverse transcriptase (7901-ahTERT) (20) or with a pCI-neo vacant vector (7901-neo) were routinely grown in RPMI-1640 medium supplemented with penicillin $(100 \mathrm{U} / \mathrm{ml})$, streptomycin $(100 \mu \mathrm{g} / \mathrm{ml})$, and $10 \%$ calf serum (CS) in a humidified chamber with an atmosphere of $95 \%$ air and $5 \% \mathrm{CO}_{2}$ at $37^{\circ} \mathrm{C}$.

For transfection, cells $\left(5 \times 10^{5} / \mathrm{ml}\right)$ were seeded $24 \mathrm{~h}$ before transfection into 24- $\mathrm{mm}^{2}$ culture dishes. SGC-7901, 7901ahTR and 7901-ahTERT cells were each stably transfected with $20 \mu \mathrm{g}$ pcDNA-aTNKS using the DOTAP lipofectin method according to the manufacturer's protocol. The cells were then treated with RMPI-1640 medium supplemented with $100 \mu \mathrm{g} / \mathrm{ml}$ Zeocin (Invitrogen) in order to select for colonies containing the pcDNA-aTNKS recombinant DNA. Selection occurred $24 \mathrm{~h}$ after transfection and lasted for 2-3 weeks. The pcDNA-aTNKS transfected cells were named 7901-aTNKS, 7901-ahTR-aTNKS, 7901-ahTERT-aTNKS and 7901-zeo respectively. Moreover, SGC-7901 cells transfected with pcDNA3.1/Zeo(-) vacant vector (7901-zeo) and 7901-neo cells served as controls.

$P C R$. In order to identify whether the exogenous gene integrated into the DNA of pcDNA-aTNKS transfected cells (7901-aTNKS, 7901-ahTR-aTNKS, 7901-ahTERT-aTNKS and 7901-zeo), we amplified the Zeocin resistance gene (ZeoR) by PCR using ZeoR specific primers (forward: 5'-GT CAGTCCTGCTCCTCGG-3' and reverse: 5'-GGCCAAGT TGACCAGTGC-3'). DNA was extracted from the above cells via standard methods. The total volume of the PCR reaction system was $25 \mu 1$, containing $0.2 \mathrm{mM}$ dNTP, $1 \mathrm{mM}$ of each primer, $2 \mathrm{U}$ Taq polymerase, $1 \mathrm{X}$ reaction buffer and $200 \mathrm{ng}$ of DNA template. Samples were subjected to 35 cycles of denaturation $\left(94^{\circ} \mathrm{C}\right.$ for $\left.1 \mathrm{~min}\right)$, annealing $\left(60^{\circ} \mathrm{C}\right.$ for $1 \mathrm{~min})$ and extension $\left(72^{\circ} \mathrm{C}\right.$ for $\left.1 \mathrm{~min}\right)$ in a PCR thermocycler (Perkin-Elmer Cetus, Norwalk, CA, USA). Ten microliters of the amplified product was analyzed by gel electrophoresis (2\% agarose) and ethidium bromide (EB) staining.

$R T-P C R$. RT-PCR was performed using the two-step RTPCR kit (Takara Co., Dalian, China). Extraction of total RNA was performed using the Tripure reagent (Roche Diagnostics, Germany). The primers and the length of amplification product are shown in Table I. The total volume of the RT-PCR reaction was $25 \mu 1$, containing $0.2 \mathrm{mM}$ dNTP, $1 \mathrm{mM}$ of each primer, $1 \mathrm{mM} \mathrm{MgSO}{ }_{4}, 0.1 \mathrm{U} / \mathrm{ml} \mathrm{AMV} \mathrm{tran-}$ scriptase, $0.1 \mathrm{U} / \mathrm{ml}$ Tf1 DNA polymerase, $1 \mathrm{X}$ reaction buffer and $100 \mathrm{ng}$ of RNA template. The amplification condition was $48^{\circ} \mathrm{C}$ for $45 \mathrm{~min}$ for retrotranscription and 40 cycles $\left(94^{\circ} \mathrm{C}, 30 \mathrm{sec} ; 60^{\circ} \mathrm{C}, 30 \mathrm{sec} ; 68^{\circ} \mathrm{C}, 7 \mathrm{~min}\right.$ ) for PCR (PerkinElmer Cetus). The amplified product was analyzed by gel electrophoresis in a $2 \%$ agarose gel and EB staining. 
Table I. Primer sequence of telomerase subunits and GAPDH.

\begin{tabular}{llc}
\hline Gene & \multicolumn{1}{c}{ Sequence } & Amplified length (bp) \\
\hline GAPDH & & 598 \\
Up & 5'CCACCCATGGCAAATTCCATGGCA3' & \\
Down & 5'TCTAGACCCCACCTCAGGTCCAC3' & 145 \\
hTERT & & 264 \\
Up & 5'CCGAAGAGTGTCTGGAGCAA3' \\
Down & 5'GGATGAACCGCACTCTGCA3' \\
TP1 & & \\
Up & & \\
Down & 5'TCAAGCGAAACCTGAATCTGAC3' \\
hTR & 5'CCCGAGTGAATCTTTCTACGC3' \\
Up & & \\
Down & 5'TCTAACCCTAACTGAGAAGGGCGTAG3' \\
\hline
\end{tabular}

Western blot analysis. Protein detection of tankyrase 1, TRF1 and TRF2 in the above cells was measured as previously reported (20). The immunoreactive bands were detected using the ECL Western blot analysis system (Amersham Biosciences, Buckinghamshire, UK).

Analysis of telomerase activity by TRAP-ELISA. Telomerase activity was measured using the TeloTAGGG Telomerase PCR ELISA kit (Roche Diagnostics). In brief, $2 \times 10^{6}$ transfected and non-transfected SGC-7901 cells were lysed, respectively in $200 \mu \mathrm{l}$ of ice-cold lysis buffer for $30 \mathrm{~min}$. The lysates were centrifuged at $12,000 \mathrm{x}$ g for $30 \mathrm{~min}$ at $4^{\circ} \mathrm{C}$, and the supernatant was rapidly frozen and stored at $-80^{\circ} \mathrm{C}$. Two microliters of each extract corresponding to $2 \times 10^{3}$ cells were assayed for telomerase activity in $50 \mu 1$ of the reaction mixture using a modified TRAP assay. Telomeric repeats were added to a biotin-labeled primer during the first telomerase-mediated extension reaction. Then, the elongation products were amplified by PCR. An aliquot of the PCR product was denatured, hybridized to a DIG-labeled, telomeric repeats-specific probe and bound to a streptavidin-coated 96-well plate. Finally, the immobilized PCR product was detected with an anti-DIG-POD antibody visualized by a color reaction product using the substrate TMB and quantified photometrically at $450 \mathrm{~nm}$.

Determination of telomere length by Southern blot analysis. Terminal restriction fragment (TRF) assays were performed by Southern blot analysis as described previously using ${ }^{32} \mathrm{P}$-labelled $(\mathrm{TTAGGG})_{4}$ probe $(11,12)$. The autoradiogram was quantitated by densitometric scanning using the SiXing BioImage System (Shanghai, China). A result of telomere length was measured as previously described $(13,14)$.

Growth curve by MTT assay. The above cells were plated onto 96-well culture dishes. At each time point, 3-(4,5Dimethylthiazol-2-yl)-2,-diphenyltetrazolium bromide (MTT), which was dissolved in PBS to a final concentration of $0.5 \mathrm{mg} / \mathrm{ml}$, was added to the cells. After $4 \mathrm{~h}$ of incubation, the cells were solubilized in $150 \mu \mathrm{l}$ dimethylsulfoxide. The intensity of the dissolved formazan crystals was measured at $490 \mathrm{~nm}$. Each experiment was repeated three times.

Statistics. The data are expressed as the mean \pm SD. Statistical analysis was performed using the Student's t-test. The difference was considered statistically significant when the p-value was $<0.05$. All statistical analysis was carried out with the SPSS 11.5 software.

\section{Results}

Construction and identification of antisense tankyrase 1 eukaryotic expression vector. Because there is an internal EcoRI site in the cDNA of tankyrase 1, part of the cDNA of tankyrase 1 , which was $3.4 \mathrm{~kb}$ in length, was digested from the T220 plasmid with the EcoRI restriction endonuclease and then inserted into the pcDNA3.1/Zeo(-) eukaryotic expression vector. The BamHI restriction endonuclease was used to determine the orientation of the recombinant DNAs. After digestion with BamHI, the sense tankyrase 1 expression vector formed two fragments (5.0 and $3.4 \mathrm{~kb}$ ), and the antisense tankyrase 1 expression vector formed two fragments (8.2 and $0.2 \mathrm{~kb}$ ). These two expression vectors were named pcDNA-sTNKS and pcDNA-aTNKS, respectively. Further sequencing was applied to verify the orientation of the recombinant vectors (data not shown).

Transfection of pcDNA-aTNKS. The recombinant pcDNAaTNKS plasmid was introduced into the SGC-7901, 7901ahTR and 7901-ahTERT cells by the DOTAP lipofectin method. After $24 \mathrm{~h}$ of transfection, $400 \mu \mathrm{g} / \mathrm{ml}$ of G418 and/ or $100 \mu \mathrm{g} / \mathrm{ml}$ of Zeocin were added into the RPMI-1640 medium. After 4 weeks of selection, individual drug resistant clones were randomly collected from the pCDNA-aTNKS 
A

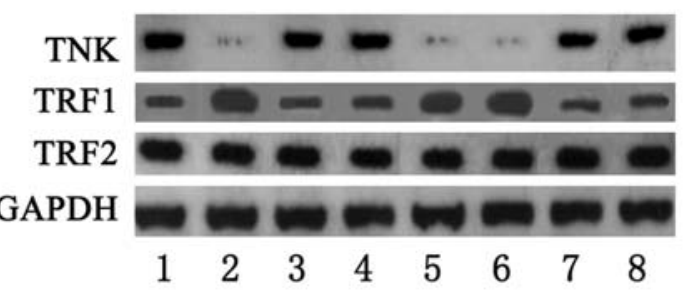

B

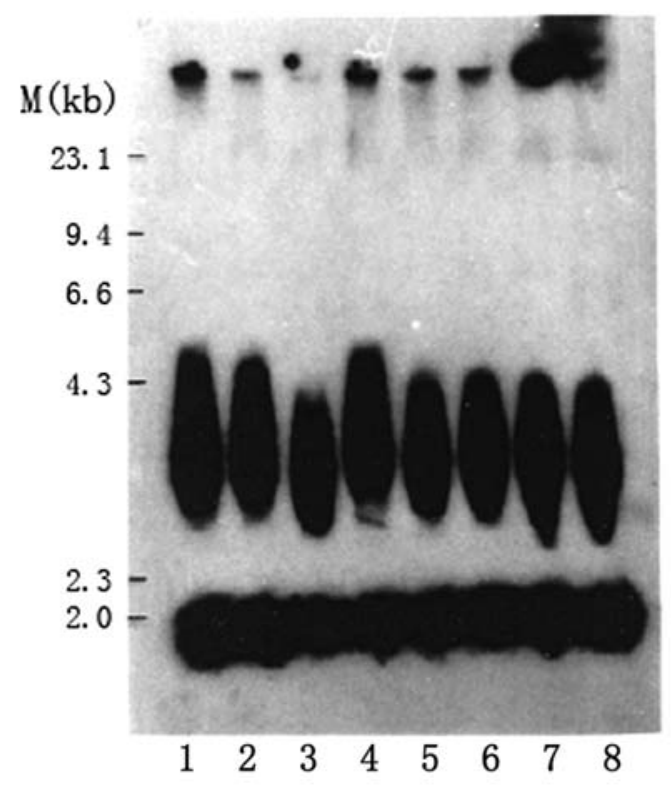

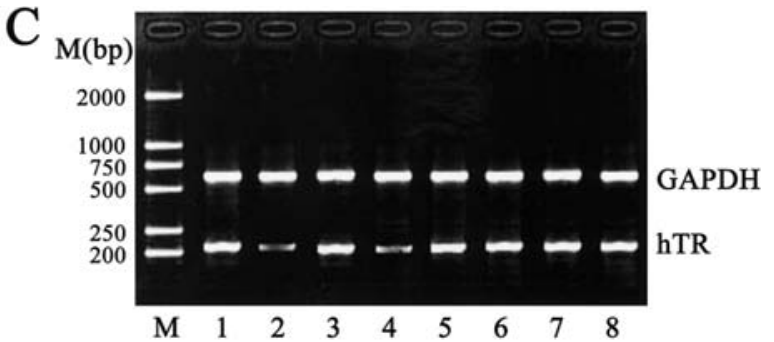
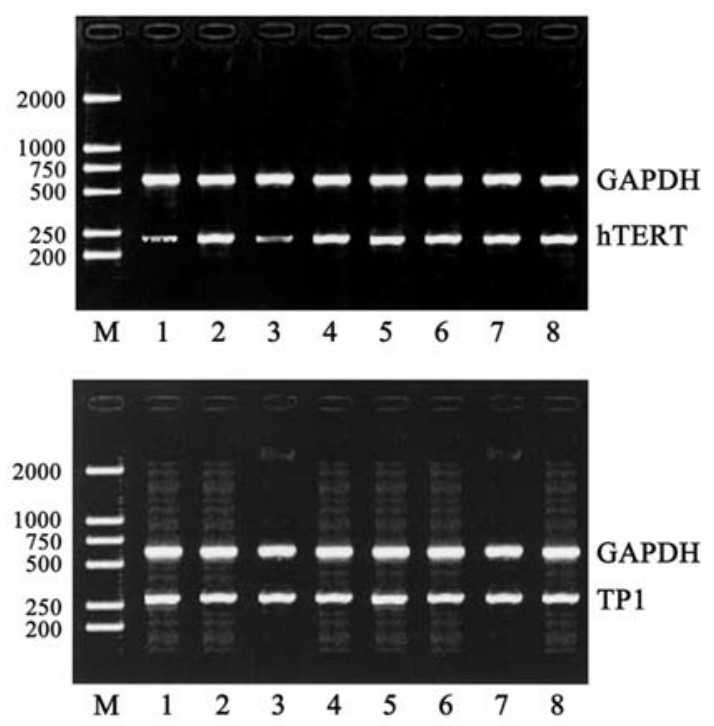

Figure 2. Determination of terminal restriction fragment (TRF) and telomerase subunits in aTNKs transfected and untransfected cells. (A) Protein expression of tankyrase 1, TRF1 and TRF2 in aTNKS by Western blot analysis. 1, SGC-7901; 2, 7901-aTNKS; 3, 7901-ahTR; 4, 7901-ahTERT; 5, 7901-ahTR-aTNKS; 6, 7901-ahTERT-aTNKS; 7, 7901-neo; 8, 7901-zeo. (B) TRF measured by Southern blot analysis. 1, SGC-7901; 2, 7901-zeo; 3, 7901-aTNKS; 4, 7901-neo; 5, 7901-ahTR; 6, 7901-ahTERT; 7, 7901-ahTR-aTNKS; 8, 7901-ahTERT-aTNKS. (C) Expression of telomerase subunits by RT-PCR. 1, PCR marker; 2, 7901-ahTERT-aTNKS; 3, 7901-ahTR-aTNKS; 4, 7901-ahTERT; 5, 7901-ahTR; 6, 7901-aTNKS; 7, 7901-neo; 8, 7901-zeo; 9, SGC-7901.

transfected cultures. As controls, drug resistant clones were selected from vacant vector pcDNA3.1/Zeo(-) transfected SGC-7901 cells. The selected clones were named 7901aTNKs, 7901-ahTR-aTNKs, 7901-ahTERT-aTNKS and 7901-zeo, respectively.

In order to determine whether the exogenous gene integrated into the DNA of pcDNA-aTNKS transfected cells, the exogenous ZeoR was amplified by PCR with ZeoR specific primers. The results showed that ZeoR, which is $357 \mathrm{bp}$ in length, could be amplified in recombinant pcDNAaTNKS or vacant pcDNA3.1/Zeo(-) vector transfected cells (7901-aTNKs, 7901-ahTR-aTNKs, 7901-ahTERT-aTNKS and 7901-zeo), but not in untransfected SGC-7901 cells. This result indicates that the exogenous gene had successfully integrated into the DNA of the above cells.

Expression of tankyrase 1. Western blot analysis showed that tankyrase 1 expression was significantly decreased in 7901aTNKS, 7901-ahTR-aTNKS and 7901-ahTERT-aTNKS cells (Fig. 2A). However, tankyrase 1 expression remained unchanged in 7901-ahTR and 7901-ahTERT cells compared with SGC-7901 cells. These results suggest that transfection of the antisense tankyrase 1 vector decreases tankyrase expression, while the transfection of the antisense hTR or antisense hTERT vectors do not affect the expression of tankyrase 1 .
Table II. Telomerase activity in aTNKs transfected and untransfected cells.

\begin{tabular}{lcc}
\hline & N & $\begin{array}{c}\text { Telomerase activity } \\
(\text { Mean } \pm \text { SD) }\end{array}$ \\
\hline SGC-7901 & 3 & $37.06 \pm 0.06$ \\
7901-zeo & 3 & $37.04 \pm 0.04^{\mathrm{a}}$ \\
7901-neo & 3 & $37.11 \pm 0.03^{\mathrm{a}}$ \\
7901-aTNKS & 3 & $37.07 \pm 0.03^{\mathrm{a}}$ \\
7901-ahTR & 3 & $21.38 \pm 0.03^{\mathrm{b}}$ \\
7901-ahTERT & 3 & $14.78 \pm 0.05^{\mathrm{c}}$ \\
7901-ahTR-aTNKS & 3 & $22.48 \pm 0.03^{\mathrm{b}}$ \\
7901-ahTERT-aTNKS & 3 & $14.82 \pm 0.04^{\mathrm{c}}$ \\
\hline
\end{tabular}

Compared with SGC-7901, ${ }^{\mathrm{a}} \mathrm{p}>0.05,{ }^{\mathrm{b}} \mathrm{p}<0.05$; Compared with SGC-7901, 7901-ahTR or 7901-ahTR-aTNKS, ${ }^{\mathrm{c}} \mathrm{p}<0.05$.

Telomerase activity in 7901-aTNKS cells. TRAP-ELISA demonstrated that there was no significant difference in telomerase activity among the three control groups (SGC7901, 7901-zeo and 7901-neo cells) ( $>>0.05)$. Compared with the above control groups, telomerase activity was 


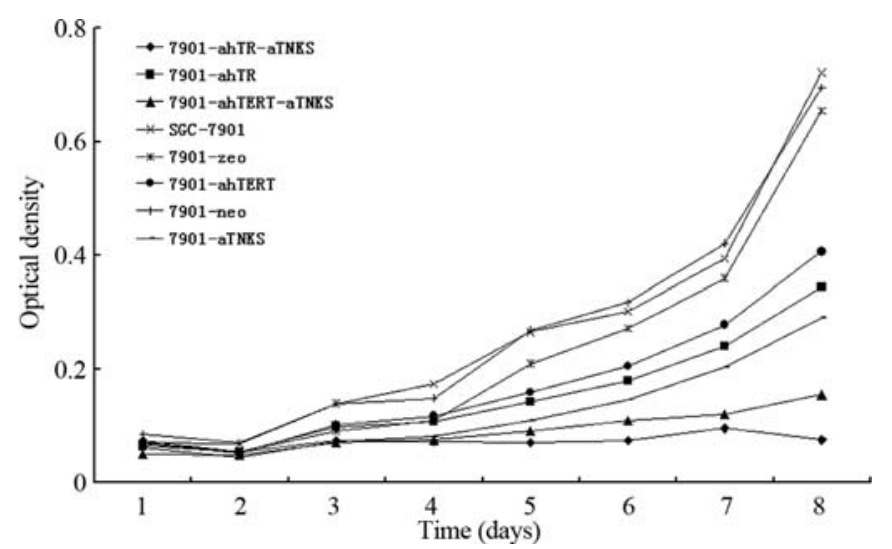

Figure 3. Growth curve for aTNKS transfected and untransfected SGC-7901 cells by MTT.

decreased by 39 and $60 \%$ in 7901-ahTR and 7901-ahTERT cells, respectively $(\mathrm{p}<0.05)$ (Table II). Moreover, telomerase activity was lower in 7901-ahTERT cells compared to 7901ahTR cells $(\mathrm{p}<0.05)$. We also found that antisense tankyrase 1 did not affect telomerase activity in SGC-7901 cells. Telomerase activity in was the same in 7901-aTNKS cells and control groups $(\mathrm{p}>0.05)$. There was also no significant difference in telomerase activity between 7901-ahTR cells and 7901-ahTR-aTNKS cells ( $\mathrm{p}>0.05)$, or between 7901ahTERT cells and 7901-ahTERT-aTNKS cells ( $\mathrm{p}>0.05)$. These results suggest that ahTR and ahTERT transfection could significantly decrease telomerase activity, while aTNKS transfection could not affect telomerase activity in SGC-7901 cells.

Terminal restriction fragment length in 7901-aTNKS cells. The terminal restriction fragment (TRF) length in SGC-7901, 7901-zeo, 7901-aTNKS, 7901-neo, 7901-ahTR, 7901ahTERT, 7901-ahTR-aTNKS and 7901-ahTERT-aTNKS cells was 3.84, 3.90, 3.12, 3.88, 3.34, 3.28, 2.98 and $2.94 \mathrm{~kb}$, respectively (Fig. 2B). This result indicated that aTNKS transfection could decrease TRF length in SGC-7901 cells. Moreover, we also found that telomere length was significantly shorter in groups receiving combined inhibition of tankyrase 1 and telomerase than by inhibition of either tankyrase 1 or telomerase alone in the human gastric cancer cell line SGC-7901.

Determination of telomerase subunits in aTNKs transfected and untransfected cells. We quantified the mRNA of telomerase subunits by semi-quantitative RT-PCR in the above eight kinds of cells. The results showed that the expression of hTERT mRNA was significantly reduced in ahTERT transfected cells, while the expression of hTR mRNA was significantly depressed in ahTR transfected cells. However, the expression of TP1 mRNA remained unchanged in all cells. We also found that aTNKS had no effect on the expression of hTERT and hTR (Fig. 2C). This result is consistent with the activity of telomerase in the above cells, which suggests that antisense tankyrase 1 does not affect telomerase activity.
Expression of telomere-binding protein TRF1 and TRF2. We further measured the protein expression of TRF1 and TRF2 in aTNKS transfected and untransfected SGC-7901 cells. The results demonstrated that the levels of TRF2 protein remained unchanged in these cells. However, the level of TRF1 protein was increased in aTNKS transfected cells (7901-aTNKS, 7901-ahTR-aTNKS and 7901-ahTERTaTNKS), but remained unchanged in the rest of the untransfected cells (Fig. 2A). This result is consistent with the telomere lengths that were detected by Southern blot analysis, which suggests that aTNKS can decrease the telomere length through increased expression of TRF1.

Growth features of transfected and untransfected cells. The average absorbance rate of the three wells, which represents the proliferative speed, was measured for eight days for each group and marked as eight-point cell growth curves. As shown in Fig. 3, the proliferation capacity was significantly inhibited in 7901-ahTR, 7901-ahTERT, 7901-aTNKS, 7901ahTR-aTNKS and 7901-ahTERT-aTNKS cells compared with control groups (SGC-7901, 7901-neo, 7901-zeo). Moreover, we also found that the average proliferative speed was slower in 7901-aTNKS cells compared to 7901-ahTR and 7901-ahTERT cells, while the average proliferative speed was slower in 7901-ahTR-aTNKS and 7901-ahTERT-aTNKS cells compared to 7901-ahTR and 7901-ahTERT cells. This result suggests that aTNKS transfection can further decrease the proliferative capacity of SGC-7901 cells.

\section{Discussion}

Tankyrase 1, as a positive regulator for telomere length, plays an important role in telomere elongation by enhancing telomerase access to telomeres. From a therapeutic perspective, tankyrase 1 is expected to be a novel target for cancer treatment. Although telomerase inhibitors shorten the length of telomeres, complete inhibition of telomerase activity is thought to be technically difficult. During progressive shortening, telomeres become more resistant to telomerase inhibitors (19). For example, shortened telomeres have fewer TRF1. This decrease in TRF1 enhances the access for any residual telomerases to act on telomeres. Because tankyrase 1 decreases the loading of TRF1 to a chromosome end, its blockade resumes telomere binding of TRF1, which in turn compromises telomere access to the residual telomerase activity. Thus, combined inhibition of tankyrase 1 and telomerase may be a rational target for telomere-directed cancer therapeutics (19).

In the present study, we studied the synergistic action of tankyrase 1 and telomerase in the regulation and maintenance of telomere length in mammalian cells. To this end, we constructed antisense tankyrase 1 (aTNKS) eukaryotic expression vectors and transfected them into SGC-7901 cells, a human gastric cancer cell line, as well as into cells which had been transfected with antisense hTR (7901-ahTR) and hTERT (7901-ahTERT) by using DOTAP liposome. The results demonstrated that telomere length was more shortened in 7901-ahTR-aTNKS and 7901-ahTERT-aTNKS cells compared to 7901-ahTR and 7901-ahTERT cells. Moreover, we also found that aTNKS transfection did not affect 
telomerase activity in SGC-7901 cells. These results indicated that transfection of aTNKS was able to induce a marked inhibition of tankyrase 1 protein and a decrease in telomere length in SGC-7901 cells.

Tankyrase 1 is an important component of the human telomeric complex. It does not contain a nuclear localization signal. Its telomeric localization is regulated by both the cell cycle and TRF1 (18). Many previous studies have shown that tankyrase 1 is able to bind to TRF1 and that it is responsible for its ribosylation, leading to the inhibition of TRF1 binding to telomere DNA. As a consequence, telomerase gains access to the telomere, allowing for telomere elongation (22). TRF1 regulates telomere length and function by at least two possible mechanisms (23). Ancelin et al demonstrated that inhibition of telomerase activity by a modified oligonucleotide did not further increase the pace of telomere erosion caused by TRF1 targeting, which suggested that telomerase itself is the target of TRF1 regulation (24). On the other hand, Smogorzewska et al (12) reported that overexpression of TRF1 in the tetracycline-responsive human fibrosarcoma cell line HTC75 results in a gradual decline in telomere length at a rate of $\sim 10 \mathrm{bp} /$ population doubling (PD). Conversely, the expression of a dominant negative allele of TRF1, which removes endogenous TRF1 from telomeres, leads to telomere elongation. As a result, Smogorzewska et al concluded that TRF1 did not affect the activity of telomerase. When telomeres elongate inappropriately, a large amount of TRF1 proteins block the telomerase-mediated elongation in order to maintain an optimal telomere length (12). In this report, we show that the expression of TRF1 increases after the inhibition of tankyrase 1, supporting the assumption that TRF1 acts in cis as a negative regulator of telomere length, independent of telomerase in SGC-7901 cells.

TRF2, which is absent from the NH2-terminal acidic domain of TRF1 that is necessary for interaction with tankyrase 1, does not interact with tankyrase 1 (13). TRF2 binds directly to the TTAGGG repeats of double-stranded telomeric DNA and contributes to the formation of the chromosomeprotecting T-loops, which is important for maintaining stable telomeres (25). We found that the inhibition of tankyrase 1 did not affect the expression of TRF2. At the same time, inhibition of telomerase by antisense hTR or HTERT transfection did not lead to any significant changes in TRF2 expression in SGC-7901 cells. These results suggested that TRF2 is independent of the tankyrase 1 and telomerase activity.

Elongation of telomeres by tankyrase 1 is dependent on telomerase activity $(26,27)$. Overexpression of tankyrase 1 in the nucleus led to the elongation of telomeres. However, this was not accompanied by altered telomerase activity (16). We showed that transfection of antisense tankyrase 1 led to telomere shortening, but had no effect on telomerase activity. These results indicated that tankyrase 1 did not act by directly modulating the expression of telomerase. Finnon et al reported that $\mathrm{X}$-irradiation transiently increased telomerase activity in two mouse myeloid leukemia cell lines in a dosedependent fashion, but did not elevate the expression level of tankyrase 1 (28). Gelmini et al also found that there was no relationship between tankyrase 1 and hTERT mRNA expression in breast cancer (29). These results showed that up-regulation or down-regulation of tankyrase 1 did not affect telomerase activity, suggesting that tankyrase 1 and telomerase do not directly interact with each other.

Here, we demonstrated that concomitant inhibition of tankyrase 1 and telomerase results in telomere shortening. Tankyrase 1 inhibition enhanced telomere shortening in 7901-ahTR and 7901-ahTERT cells, suggesting that cotransfection of antisense tankyrase 1 and antisense hTR or antisense hTERT may play a synergetic role in decreasing telomere length. Because shortened telomeres increase the efficiency of telomere elongation (30-32), telomere shortening may also result in the resistance of cells to telomerase inhibition. Our results showed that inhibition of tankyrase 1 reversed the resistance to telomerase inhibitors. This was consistent with the results of Seimiya et al (19), who reported that PARP inhibitors $3 \mathrm{AB}$ and PJ-34 enhanced telomere shortening induced by the MST-312 telomerase inhibitor in telomerase-positive cells but not in normal or telomerasenegative cells. We speculated that the mechanism behind the observed synergy could be the result of the inhibition of tankyrase 1 on the resistance to telomerase inhibition.

In summary, our results reveal that inhibition of tankyrase 1 could shorten telomere length and play a synergistic role with telomerase inhibitors in telomere length shortening in the gastric cancer cell line SGC-7901. Co-inhibition of tankyrase 1 and telomerase activity may be a rational strategy for telomere-directed gastric cancer therapeutics.

\section{Acknowledgements}

We thank Professor Susan Smith and Titia de Lange from Rockefeller University, New York, USA, for generously providing the T220 plasmid that contained the full-length cDNA of tankyrase 1 . This study was supported by grants from the Chongqing Science Fund for Distinguished Young Scholars (CSTC, 2009BA5045) and the National Basic Research Program (973 Program) (No. 2010CB529400).

\section{References}

1. Blackburn EH: Structure and function of telomeres. Nature 350: 569-573, 1991.

2. Greider CW: Telomere length regulation. Annu Rev Biochem 65: 337-365, 1996

3. Muniyappa K and Kironmai KM: Telomere structure, replication and length maintenance. Crit Rev Biochem Mol Biol 33: 297-336, 1998.

4. Dahse R, Fiedler W and Ernst G: Telomeres and telomerase: biological and clinical importance. Clin Chem 43: 708-714, 1997.

5. Feng J, Funk WD, Wang SS, et al: The RNA component of human telomerase. Science 269: 1236-1241,1995.

6. Harrington L, McPhail T, Mar V, et al: A mammalian telomeraseassociated protein. Science 275: 973-977, 1997.

7. Nakamura TM, Morin GB, Chapman KB, et al: Telomerase catalytic subunit homologs from fission yeast and human. Science 277: 955-959, 1997.

8. Kim NW, Piatyszek MA, Prowse KR, et al: Specific association of human telomerase activity with immortal cells and cancer. Science 266: 2011-2015, 1994.

9. Shay JW and Bacchetti S: A survey of telomerase activity in human cancer. Eur J Cancer 33: 787-791, 1997.

10. Cook BD, Dynek JN, Chang W, et al: Role for the related poly(ADP-Ribose) polymerases tankyrase 1 and 2 at human telomeres. Mol Cell Biol 22: 332-342, 2002.

11. Blasco MA: Mammalian telomeres and telomerase: why they matter for cancer and aging. Eur J Cell Biol 82: 441-446, 2003. 
12. Smogorzewska A, van Steensel B, Bianchi A, et al: Control of human telomere length by TRF1 and TRF2. Mol Cell Biol 20: 1659-1668, 2000.

13. Smith S, Giriat I, Schmitt A and de Lange T: Tankyrase, a poly(ADP-ribose) polymerase at human telomeres. Science 282: 1484-1487, 1998.

14. Van Steensel B and de Lange T: Control of telomere length by the human telomeric protein TRF1. Nature 385: 740-743, 1997.

15. Karlseder J, Smogorzewska A and de Lange T: Senescence induced by altered telomere state, not telomere loss. Science 295: 2446-2449, 2002.

16. Smith $\mathrm{S}$ and de Lange T: Tankyrase promotes telomere elongation in human cells. Curr Biol 10: 1299-1302, 2000.

17. Seimiya $\mathrm{H}$ and Smith $\mathrm{S}$ : The telomeric poly(ADP-ribose) polymerase, tankyrase 1 , contains multiple binding sites for telomeric repeat binding factor 1 (TRF1) and a novel acceptor, 182-kDa tankyrase-binding protein (TAB182). J Biol Chem 277: 14116-14126, 2002.

18. Smith $\mathrm{S}$ and de Lange T: Cell cycle dependent localization of the telomeric PARP, tankyrase, to nuclear pore complexes and centrosomes. J Cell Sci 112: 3649-3656, 1999.

19. Seimiya H, Muramatsu Y, Ohishi T, et al: Tankyrase 1 as a target for telomere-directed molecular cancer therapeutics. Cancer Cell 7: 25-37, 2005.

20. Yang SM, Fang DC, Yang JL, et al: Antisense human telomerase reverse transcriptase could partially reverse malignant phenotypes of gastric carcinoma cell line in vitro. Eur J Cancer Prev 17: 209-217, 2008

21. Yang SM, Fang DC, Yang JL, et al: Effect of antisense human telomerase RNA on malignant phenotypes of gastric carcinoma. J Gastroenterol Hepatol 17: 1144-1152, 2002.

22. Greider CW: Telomeres do D-loop-T-loop. Cell 97: 419-422, 1999.
23. Okamoto K, Iwano $\mathrm{T}$, Tachibana M, et al: Distinct roles of TRF1 in the regulation of telomere structure and lengthening. J Biol Chem 283: 23981-23988, 2008.

24. Ancelin K, Brunori M, Bauwens S, et al: Targeting assay to study the cis functions of human telomeric proteins: evidence for inhibition of telomerase by TRF1 and for activation of telomere degradation by TRF2. Mol Cell Biol 22: 3474-3487, 2002.

25. Van Steensel B, Smogorzewska A and de Lange T: TRF2 protects human telomeres from end-to-end fusions. Cell 92: 401-413, 1998.

26. Chang W, Dynek JN and Smith S: TRF1 is degraded by ubiquitin-mediated proteolysis after release from telomeres. Genes Dev 17: 1328-1333, 2003.

27. Hanahan D and Weinberg RA: The hallmarks of cancer. Cell 100: 57-70, 2000

28. Finnon P, Silver AR and Bouffler SD: Upregulation of telomerase activity by $\mathrm{X}$-irradiation in mouse leukaemia cells is independent of Tert, Terc, Tnks and Myc transcription. Carcinogenesis 21: 573-578, 2000

29. Hemann MT, Strong MA, Hao LY, et al: The shortest telomere, not average telomere length, is critical for cell viability and chromosome stability. Cell 107: 67-77, 2001.

30. Gelmini S, Poggesi M, Distante V, et al: Tankyrase, a positive regulator of telomere elongation, is overexpressed in human breast cancer. Cancer Lett 216: 81-87, 2004.

31. Marcand S, Brevet V and Gilson E: Progressive cis-inhibition of telomerase upon telomere elongation. EMBO J 18: 3509-3519, 1999.

32. Ouellette MM, Liao M, Herbert BS, et al: Subsenescent telomere lengths in fibroblasts immortalized by limiting amounts of telomerase. J Biol Chem 275: 10072-10076, 2000. 